\title{
Torque de inserção e resistência ao arrancamento dos parafusos vertebrais com alma cilíndrica e cônica*
}

\author{
Insertion torque and pullout strength of vertebral \\ screws with cylindrical and conic core
}

\author{
Ariane Zamarioli' ${ }^{1}$ Priscila Angelotti Simões ${ }^{2}$, Antônio Carlos Shimano ${ }^{3}$, Helton L.A. Defino4
}

\section{RESUMO}

Objetivo: Avaliar o torque de inserção e a força de arrancamento de três diferentes parafusos utilizados para a fixação anterior da coluna vertebral, considerando a influência do diâmetro do orifíciopiloto (DOP), a densidade dos corpos de prova (CDP)

* Trabalho de Dissertação de Mestrado realizado no Laboratório de Bioengenharia do Departamento de Biomecânica, Medicina e Reabilitação do Aparelho Locomotor da Faculdade de Medicina de Ribeirão Preto da Universidade de São Paulo - USP - Ribeirão Preto (SP), Brasil.

Trabalho realizado com apoio da Fundação de Amparo à Pesquisa do Estado de São Paulo (FAPESP) e Coordenação de Aperfeiçoamento de Pessoal de Nível Superior (CAPES).

1. Acadêmica do Curso de Pós-Graduação da Área Ortopedia, Traumatologia e Reabilitação do Aparelho Locomotor - Faculdade de Medicina de Ribeirão Preto da Universidade de São Paulo - USP - Ribeirão Preto (SP), Brasil.

2. Acadêmica do Curso de Pós-Graduação da Área Ortopedia, Traumatologia e Reabilitação do Departamento de Biomecânica, Medicina e Reabilitação da Faculdade de Medicina de Ribeirão Preto da Universidade de São Paulo - USP - Ribeirão Preto (SP), Brasil.

3. Doutor, Professor do Departamento de Biomecânica, Medicina e Reabilitação do Aparelho Locomotor - Faculdade de Medicina de Ribeirão Preto da Universidade de São Paulo - USP - Ribeirão Preto (SP), Brasil.

4. Livre-docente, Professor Titular do Departamento de Biomecânica, Medicina e Reabilitação do Aparelho Locomotor - Faculdade de Medicina de Ribeirão Preto da Universidade de São Paulo - USP - Ribeirão Preto (SP), Brasil.

Endereço para correspondência: Av. Bandeirantes, 3.900, 11o andar, Monte Alegre - 114048-900 - Ribeirão Preto (SP), Brasil. Tel./ fax: (16) 3633-0336. E-mail: hladefin@ @mrp.usp.br

Recebido em 28/8/08. Aprovado para publicação em 30/10/08.

Copyright RBO2008 e o desenho da rosca dos parafusos. Métodos: Foram utilizados CDP de poliuretana com duas densidades: 0,16 e $0,32 \mathrm{~g} / \mathrm{cm}^{3}$, e três tipos de parafusos (USS I, USS II posterior e USS II anterior). Na primeira etapa o orifício-piloto foi feito com sonda de $3,8 \mathrm{~mm}$ para todos os parafusos; na segunda etapa, com sonda menor do que o diâmetro interno dos parafusos $(3,5 \mathrm{~mm}$ para os parafusos USS I; $3,4 \mathrm{~mm}$ para os parafusos USS II posterior e $3,0 \mathrm{~mm}$ para os parafusos USS II anterior). Foram formados 12 grupos experimentais com dez corpos de prova em cada grupo, de acordo com a densidade da poliuretana, DOP e tipo de parafuso utilizado. $O$ torque foi mensurado durante a inserção dos parafusos e a força de arrancamento por meio de ensaio mecânico em máquina universal de teste. Resultados: $O$ torque máximo de inserção apresentou valores decrescentes nos corpos de prova de $0,16 \mathrm{~g} / \mathrm{cm}^{3} \mathrm{e} 0,32 \mathrm{~g} / \mathrm{cm}^{3} \mathrm{e}$ em todos os diâmetros do orifício-piloto. A força máxima de arrancamento, nos corpos de prova de $0,16 \mathrm{~g} / \mathrm{cm}^{3}$ e diâmetro da perfuração de $3,8 \mathrm{~mm}$, foi maior nos parafusos USS II posterior que nos parafusos USS I. Com o diâmetro da perfuração menor do que o diâmetro interno do parafuso, os parafusos USS II posterior e anterior apresentaram valores maiores que o parafuso USS I. Nos corpos de prova com $0,32 \mathrm{~g} / \mathrm{cm}^{3}$ de densidade e diâmetro de perfuração de $3,8 \mathrm{~mm}$, a força de arrancamento do parafuso USS II posterior e a do USS I foram maiores que a do parafuso USS II anterior. Com o diâmetro da perfuração menor que o diâmetro interno, os valores foram decrescentes entre o USS II poste- 
rior, USS II anterior e USS I. Conclusões: O torque de inserção e a força de arrancamento dos parafusos utilizados para fixação anterior da coluna vertebral são influenciados pela densidade do corpo de prova, desenho da rosca do parafuso e diâmetro do orifício-piloto.

Descritores - Coluna vertebral; Parafusos ósseos; Biomecânica; Dispositivos de fixação ortopédica

\section{ABSTRACT}

Objective: To evaluate insertion torque and pullout strength of three different screws used in the anterior fixation of the spine, considering the influence of the diameter of the pilot hole (DOP), the test specimen density (CDP), and the screw thread design. Methods: The authors used polyurethane test specimens with two densities: 0.16 and $0.32 \mathrm{~g} / \mathrm{cm}^{3}$, and three types of screws (USS I, USS II posterior, and USS II anterior). In the first stage, the pilot hole was made with a $3.8 \mathrm{~mm}$ probe for all screws; in the second stage, with a probe smaller than the inner diameter of the screws $(3.5 \mathrm{~mm}$ for screws USS I; $3.4 \mathrm{~mm}$ for USS II posterior screws, and $3.0 \mathrm{~mm}$ for USS II anterior screws). 12 experimental groups were formed with ten specimen tests in each group, according to the polyurethane density, DOP, and the type of screw used. Torque was measured during insertion of the screws and the pullout strength by a mechanical assay in a universal test machine. Results: The maximum insertion torque presented a decreasing value in test specimens of 0.16 $\mathrm{g} / \mathrm{cm}^{3}$ and $0.32 \mathrm{~g} / \mathrm{cm}^{3}$ and in all pilot hole diameters. Maximum pullout strength in test specimens of $0.16 \mathrm{~g} /$ $\mathrm{cm}^{3}$ and $3.8 \mathrm{~mm}$ of perforation diameter was greater in USS II posterior screws than in USS I screws. With the perforation diameter smaller than the inner screw diameter, USS II posterior and anterior screws presented higher values than the USS I screw. In test specimens with $0.32 \mathrm{~g} / \mathrm{cm}^{3}$ of density and perforation diameter of $3.8 \mathrm{~mm}$, the pullout strength of USS II posterior and USS I screws was greater than that of USS II anterior screws. With the smaller perforation diameter than the inner diameter, the values were decreasing between the USS II posterior screw, then the USS II anterior screw, and then the USS I screw.
Conclusion: Insertion torque and pullout strength of the screws used in the anterior fixation of the vertebral spine are influenced by the test specimen density, by the screw thread design, and by the diameter of the pilot hole.

Keywords - Spine; Bone screws; Biomechanics; Orthopedic fixation devices

\section{INTRODUÇÃO}

A instrumentação anterior para correção da escoliose foi introduzida por Dwyer et al, em 1969(1). A técnica tem sido aperfeiçoada e grande variedade de sistemas de fixação vertebral foi desenvolvida utilizando parafusos como elemento de ancoragem no corpo vertebral.

A resistência dos parafusos ao arrancamento evita sua soltura durante as manobras de correção e tem sido observada principalmente nas vértebras localizadas nas extremidades das curvas. A resistência ao arrancamento dos parafusos é multifatorial e está relacionada com a densidade mineral óssea, com o desenho do implante e com o preparo do orifício-piloto. A resistência dos implantes ao arrancamento pode ser avaliada por meio de parâmetros mecânicos como o torque de inserção e a força de arrancamento( ${ }^{(2)}$.

Modificações no desenho da rosca dos parafusos podem alterar consideravelmente a força de seu arrancamento $^{(3)}$. Parafusos com a parte externa ou com a parte interna (alma) com forma cônica foram desenvolvidos para aumentar sua resistência ao arrancamento $^{(3-5)}$.

A primeira geração de parafusos do sistema de fixação vertebral USS, (Universal Spinal System) $\left(\right.$ Synthes $\left.^{\circledR}\right)$ foi desenvolvida para ser utilizada como parafuso universal, para ser colocado no pedículo ou no corpo vertebral. Posteriormente, foi desenvolvido o parafuso USS II posterior, que também pode ser utilizado tanto para a fixação pedicular posterior como para a fixação anterior. O parafuso USS II anterior foi desenvolvido especificamente para ser utilizado na fixação anterior e possui desenho de rosca para atender às características do osso esponjoso do corpo vertebral.

O parafuso USS I possui diâmetro interno e externo com forma cilíndrica, sendo os dois diâmetros cons- 
tantes ao longo do corpo do parafuso. Os parafusos USS II possuem diâmetro interno de forma cônica e diâmetro externo de forma cilíndrica - o diâmetro externo é constante ao longo do parafuso e o diâmetro interno diminui da parte proximal em direção à ponta do parafuso.

Este estudo foi delineado considerando a importância da resistência ao arrancamento dos parafusos utilizados na fixação anterior da coluna vertebral. Seu objetivo foi avaliar o torque de inserção e força de arrancamento de três diferentes parafusos utilizados para a fixação anterior da coluna vertebral, considerando a densidade do corpo de prova, o diâmetro do orifíciopiloto e o desenho da rosca dos parafusos.

\section{MÉTODOS}

Este estudo foi desenvolvido no Laboratório de Bioengenharia da Faculdade de Medicina de Ribeirão Preto da Universidade de São Paulo (USP).

Três diferentes parafusos de titânio com $30 \mathrm{~mm}$ de comprimento e utilizados para a fixação anterior da coluna torácica e lombar foram selecionados para o estudo: USS I, USS II posterior e USS II anterior. As características dos parafusos estão detalhadas e ilustradas na tabela 1 e na figura 1.

Devido à alta variabilidade dos modelos biológicos, este estudo foi realizado com material sintético para garantir o controle sobre as variáveis, enfatizando a comparação entre os diferentes parafusos, tendo sido utilizados corpos de prova de poliuretana. Modelos similares foram utilizados em estudos para simular a resposta do osso trabecular submetido à inserção de diferentes tipos de parafusos $^{(2-3,6-11)}$.

TABELA 1

Características dos parafusos utilizados

\begin{tabular}{lccc}
\hline Medidas (mm) & USS I & $\begin{array}{c}\text { USS II } \\
\text { posterior }\end{array}$ & $\begin{array}{c}\text { USS II } \\
\text { anterior }\end{array}$ \\
\hline Diâmetro externo & 6,0 & 6,2 & 6,2 \\
Diâmetro interno & 4,80 & 5,84 (máximo) & 3,98 (máximo) \\
Comprimento & 30,0 & 30,0 & (mínimo) \\
Passo de rosca & 1,75 & 2,00 & 30,0 \\
\hline
\end{tabular}

USS - Universal Spinal System

Rev Bras Ortop. 2008;43(10):452-9

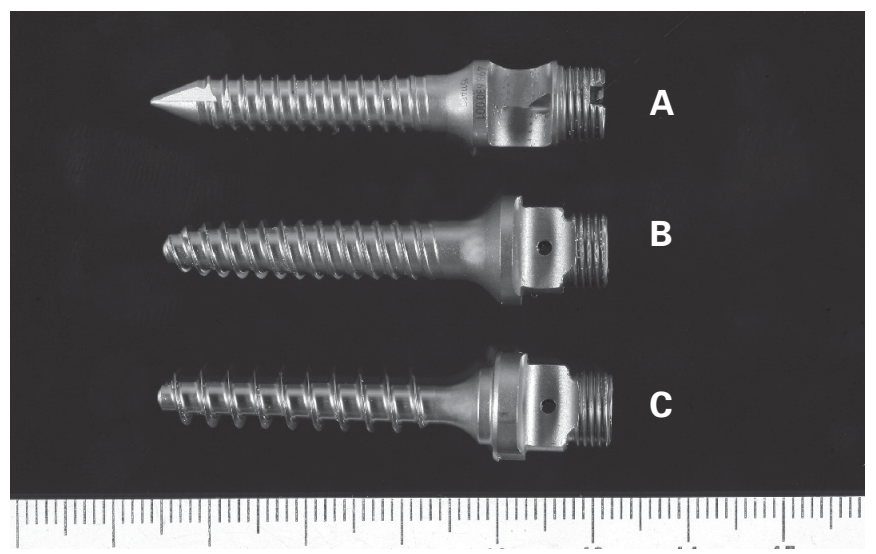

Figura 1 - Parafusos utilizados no estudo. Parafuso cilíndrico USS I com diâmetro externo de $6,0 \mathrm{~mm}$ e interno de $4,8 \mathrm{~mm}$ (A), parafuso cônico USS II posterior com diâmetro externo de $6,2 \mathrm{~mm}$ e interno variando de 3,40 a $5,84 \mathrm{~mm}$ (B) e parafuso cônico USS II anterior com diâmetro externo de $6,2 \mathrm{~mm}$ e interno variando de 3,00 a $3,98 \mathrm{~mm}$ (C)

Fonte: FMRP-USP

Blocos de poliuretana (Empresa Nacional Ossos ${ }^{\circledR}$, Jaú, SP) com $42 \mathrm{~mm}$ x $24 \mathrm{~mm}$ x $24 \mathrm{~mm}$ e de duas diferentes densidades $\left(0,16 \mathrm{~g} / \mathrm{cm}^{3} \mathrm{e} 0,32 \mathrm{~g} / \mathrm{cm}^{3}\right)$ foram utilizados para a colocação dos parafusos e mensuração dos valores do torque de inserção e da resistência ao arrancamento. Os 12 grupos experimentais foram formados de acordo com a densidade da poliuretana, diâmetro do orifício-piloto e desenho do parafuso. Cada grupo experimental foi formado por 10 corpos de prova.

Foram utilizados 120 blocos de poliuretana - $60 \mathrm{com}$ densidade de $0,16 \mathrm{~g} / \mathrm{cm}^{3}$ e 60 com densidade de $0,32 \mathrm{~g} /$ $\mathrm{cm}^{3}$.

O modelo experimental do estudo consistiu no preparo do orifício-piloto, colocação do parafuso acompanhado do torque de inserção e realização do ensaio mecânico de arrancamento. $\mathrm{O}$ estudo foi realizado em duas etapas. Na primeira etapa, o orifício-piloto foi realizado com sonda de $3,8 \mathrm{~mm}$ e, na segunda etapa, com sondas cujo diâmetro variou de acordo com o diâmetro interno dos parafusos. Para o parafuso USS I, foi utilizada sonda de $3,5 \mathrm{~mm}$ de diâmetro; para o parafuso USS II posterior, sonda de $3,4 \mathrm{~mm}$; e para o parafuso USS II anterior, sonda de $3,0 \mathrm{~mm}$. A sonda de $3,5 \mathrm{~mm}$ utilizada para o parafuso USS I corresponde a valor de diâmetro interno inferior ao do diâmetro interno do parafuso. As sondas de 3,4 e 3,0mm correspondem ao 


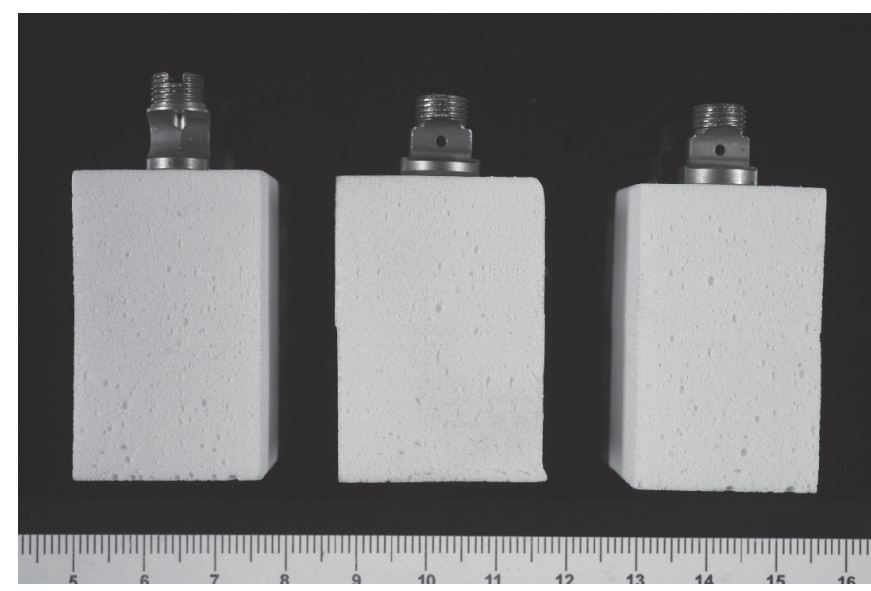

Figura 2 - Blocos de poliuretana com os parafusos inseridos: USS I (A), USS II posterior (B) e USS II anterior (C).

Fonte: FMRP-USP

menor valor de diâmetro interno dos parafusos USS II posterior e USS II anterior, respectivamente.

Cada grupo experimental era formado por 10 corpos de prova, de acordo com a densidade da poliuretana, diâmetro do orifício-piloto e tipo de parafuso utilizado.

O torque de inserção dos parafusos foi mensurado por meio de torquímetro da marca Mackena ${ }^{\circledR}$, modelo MK-2001, com capacidade de medida de torque de até 10 N.m e precisão de 0,01 N.m. O valor de torque de inserção foi mensurado a cada volta do parafuso, tendo sido considerado o valor máximo obtido, o torque máximo de inserção. A figura 2 ilustra os corpos de prova com os três diferentes tipos de parafusos.

Os testes de tração axial (arrancamento) foram realizados usando máquina universal de ensaios $\left(E M I C^{\circledR}\right.$ DL $10000 \mathrm{~N}$ ). A célula de carga utilizada para os ensaios mecânicos apresentava capacidade de medir até 2000 e 10000 N. Os ensaios foram realizados com velocidade constante de $2 \mathrm{~mm} / \mathrm{min}$ com carga máxima de 1800 e $9800 \mathrm{~N}$ ou máxima deformação de $50 \mathrm{~mm}$. Em todos os ensaios foi utilizada pré-carga de $50 \mathrm{~N}$ com tempo de acomodação de 10 segundos.

Para a realização do ensaio mecânico de arrancamento, um acessório especial foi confeccionado para permitir a conexão entre a cabeça do parafuso e a base móvel da máquina de ensaios. O corpo de prova com o parafuso inserido foi posicionado na parte inferior de
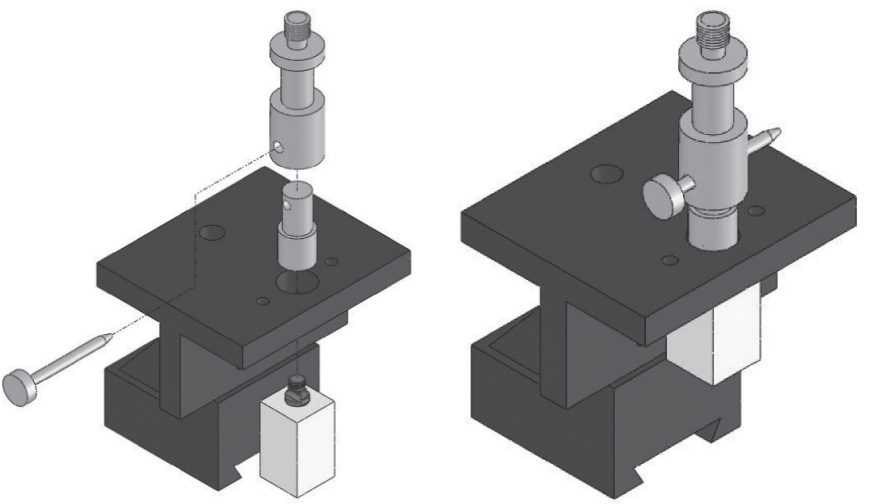

Figura 3 - O desenho esquemático ilustra a montagem dos acessórios utilizados nos ensaios mecânicos de arrancamento. $\mathrm{O}$ parafuso inserido no bloco de poliuretana é rosqueado ao acessório de conexão. O bloco de poliuretana é posicionado na parte inferior da base fixa. $\mathrm{O}$ acessório superior, fixado ao acessório de conexão através de um pino, é conectado à base móvel da máquina que realizará os ensaios de arrancamento.

Fonte: FMRP-USP

uma base fixa de aço com um orifício no centro. O acessório foi conectado ao parafuso por meio de roscas presentes nas duas estruturas e em seguida conectado à base móvel da máquina através de um pino (figura 3).

A análise dos resultados foi realizada por meio da curva força $\mathrm{x}$ deslocamento obtida durante a realização dos testes mecânicos através do programa Tesc 1.13. A propriedade mecânica avaliada foi a força máxima de arrancamento. A força máxima de arrancamento foi definida como o maior valor da curva carga versus deformação. Os resultados do torque de inserção e da força máxima de arrancamento foram submetidos ao teste de normalidade através da análise de variância multifatorial (ANOVA multifatorial). Para a comparação entre os grupos foi utilizado o pós-teste de Tukey. Em todas as análises foi adotado o nível de significância de $5 \%(\mathrm{p} \leq 0,05)$. Foi utilizado nas análises o procedimento PROC GLM do software SAS 9.0.

\section{RESULTADOS}

Torque de inserção - O TMI apresentou valores decrescentes nos parafusos USS II posterior em relação aos parafusos USS II anterior, e esses em relação aos parafusos USS I, com diferença estatística $(p<0,01)$. Essa diferença foi observada nas duas densidades dos 
corpos de prova de poliuretana e nas duas etapas do estudo (orifício-piloto com diâmetro de $3,8 \mathrm{~mm}$ e diâmetro menor que a alma do parafuso) $(\mathrm{p}<0,01)$ (gráfico 1 e tabela 2).

Força de arrancamento - A força máxima de arrancamento (FMA) dos parafusos utilizados no estudo apresentou variações de acordo com a densidade dos corpos de prova e diâmetro de perfuração do orifíciopiloto.

Nos corpos de prova com densidade de $0,16 \mathrm{~g} / \mathrm{cm}^{3} \mathrm{e}$ com orifício-piloto de $3,8 \mathrm{~mm}$, os parafusos USS II posterior apresentaram maior FMA que os parafusos USS I, com diferença estatística $(\mathrm{p}<0,01)$. Nos corpos de prova com densidade de $0.32 \mathrm{~g} / \mathrm{cm}^{3}$ e orifício-piloto de $3,8 \mathrm{~mm}$, os parafusos USS II posterior e USS I apresentaram maior resistência ao arrancamento que os parafusos USS II anterior, com diferença estatística ( $\mathrm{p}<$ 0,01) (gráfico 2).

Nos corpos de prova com densidade de $0,32 \mathrm{~g} / \mathrm{cm}^{3} \mathrm{e}$ orifício-piloto menor que o diâmetro interno do parafuso, os parafusos USS II posterior e USS II anterior apresentaram maior FMA que os parafusos USS I, com diferença estatística $(\mathrm{p}<0,01)$. Nos corpos de prova com densidade de $0,32 \mathrm{~g} / \mathrm{cm}^{3}$ e orifício-piloto menor que o diâmetro interno do parafuso, a FMA apresentou valores decrescentes nos parafusos USS II posterior em relação aos parafusos USS II anterior, e esses em relação aos parafusos USS I, com diferença estatística $(\mathrm{p}<0,01)$ (gráfico 3 e tabela 2).
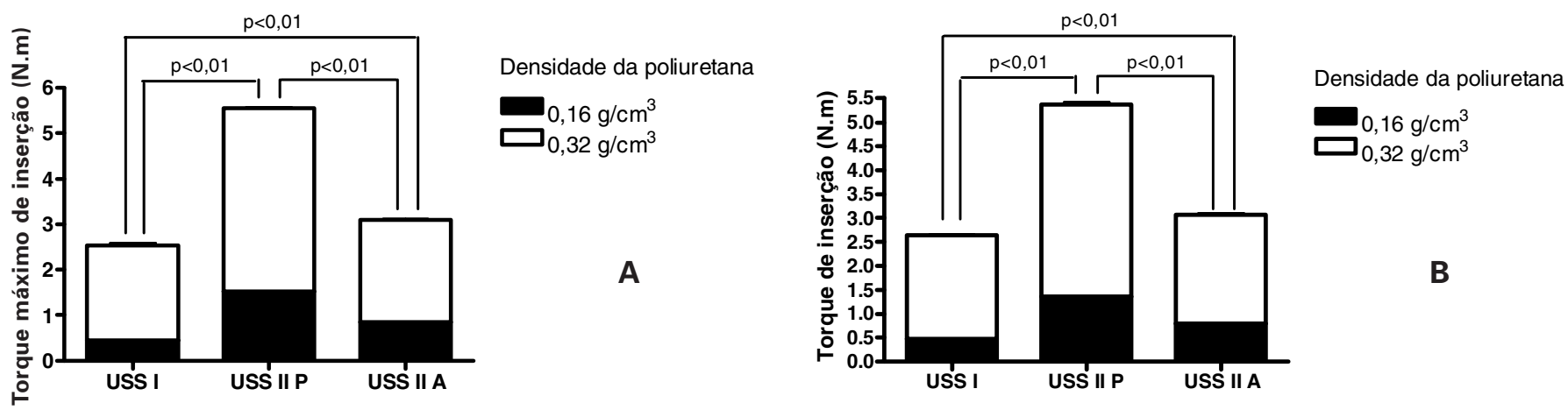

Gráfico 1 - Média e desvio-padrão do torque máximo de inserção dos parafusos nas duas densidades da poliuretana. Com sonda de $3,8 \mathrm{~mm}$ (A) e com sonda menor que o diâmetro interno dos parafusos (B).

TABELA 2

Resultados dos ensaios mecânicos nas duas densidades de poliuretana $\left(0,16 \mathrm{e} 0,32 \mathrm{~g} / \mathrm{cm}^{3}\right)$ realizados com sonda de $3,8 \mathrm{~mm}$ e com menor que o diâmetro interno dos parafusos

\begin{tabular}{|c|c|c|c|c|c|c|c|}
\hline \multirow{2}{*}{$\begin{array}{l}\text { Densidade } \\
\left(\mathrm{g} / \mathrm{cm}^{3}\right)\end{array}$} & & \multicolumn{2}{|c|}{ USS I } & \multicolumn{2}{|c|}{ USS II posterior } & \multicolumn{2}{|c|}{ USS II anterior } \\
\hline & & $3,8 \mathrm{~mm}$ & $3,5 \mathrm{~mm}$ & $3,8 \mathrm{~mm}$ & $3,4 \mathrm{~mm}$ & $3,8 \mathrm{~mm}$ & $3,0 \mathrm{~mm}$ \\
\hline \multirow{2}{*}{0,16} & $\begin{array}{l}\text { Torque de } \\
\text { inserção }\end{array}$ & $0,46 \pm 0,01$ & $0,47 \pm 0,02$ & $1,54 \pm 0,06$ & $1,36 \pm 0,08$ & $0,85 \pm 0,04$ & $0,80 \pm 0,03$ \\
\hline & $\begin{array}{l}\text { Força de } \\
\text { arrancamento }\end{array}$ & $439,19 \pm 18,03$ & $444,17 \pm 35,74$ & $488,59 \pm 36,05$ & $508,77 \pm 27,47$ & $463,14 \pm 47,53$ & $480,51 \pm 29,05$ \\
\hline \multirow{2}{*}{0,32} & $\begin{array}{l}\text { Torque de } \\
\text { inserção }\end{array}$ & $2,08 \pm 0,12$ & $2,18 \pm 0,03$ & $4,01 \pm 0,05$ & $4,01 \pm 0,15$ & $2,24 \pm 0,08$ & $2,27 \pm 0,09$ \\
\hline & $\begin{array}{l}\text { Força de } \\
\text { arrancamento }\end{array}$ & $1531,25 \pm 41,89$ & $1533,02 \pm 58,53$ & $1535,66 \pm 116,04$ & $1828,55 \pm 120,11$ & $1277,96 \pm 240,25$ & $1545,28 \pm 102,23$ \\
\hline
\end{tabular}

Fonte: FMRP-USP

USS - Universal Spinal System 


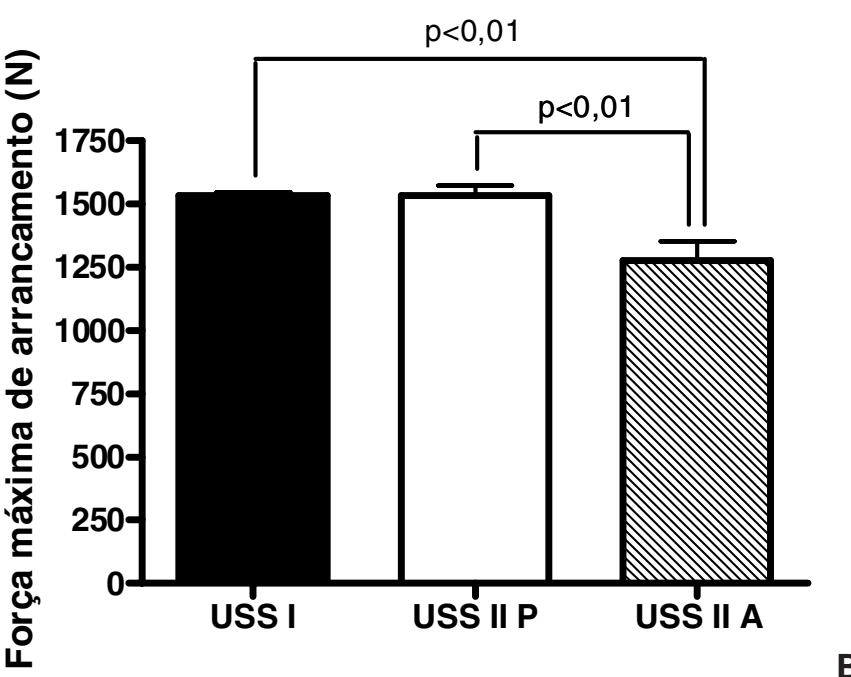

Gráfico 2 - Média e desvio-padrão da força máxima de arrancamento dos parafusos com orifício-piloto de 3,8mm. Na densidade de $0,16 \mathrm{~g} / \mathrm{cm}^{3}(A)$ e na de $0,32 \mathrm{~g} / \mathrm{cm}^{3}(B)$.
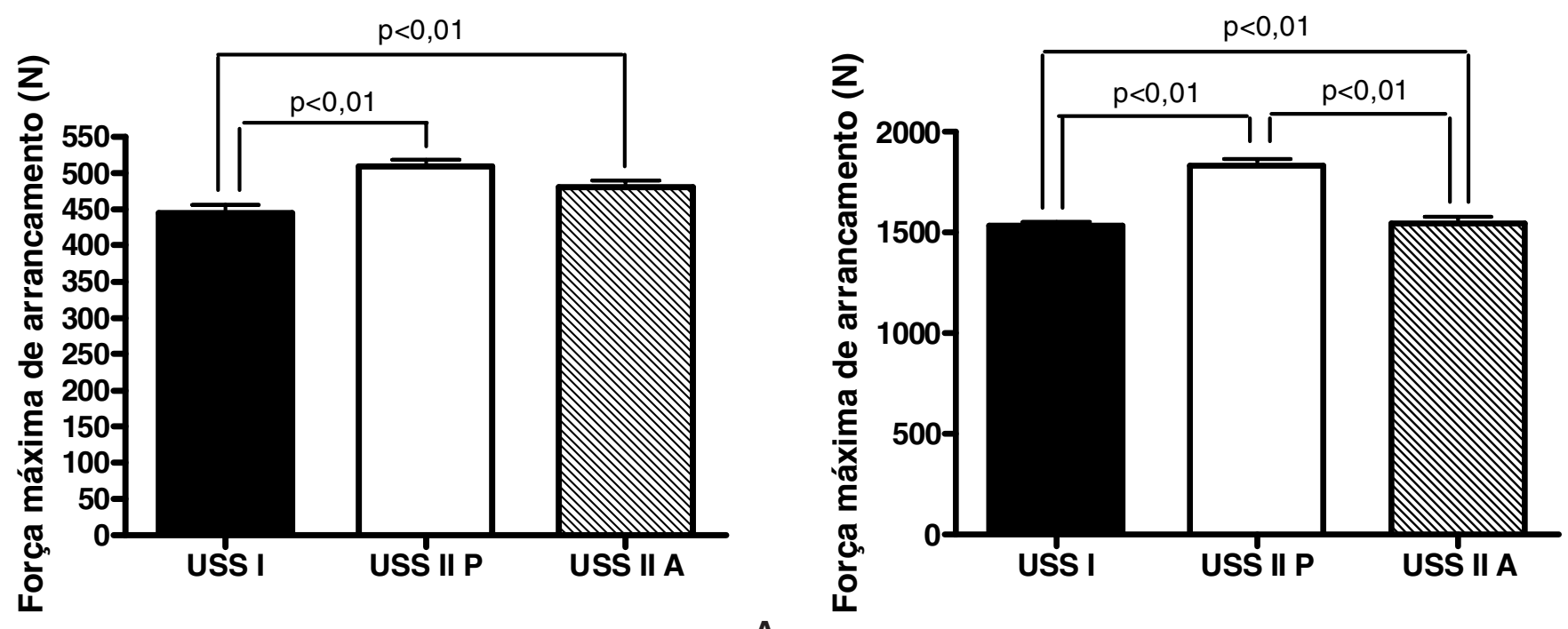

A

Gráfico 3 - Média e desvio-padrão da força máxima de arrancamento dos parafusos com orifício-piloto menor que o diâmetro interno dos parafusos. Na densidade de $0,16 \mathrm{~g} / \mathrm{cm}^{3}$ (A) e na de $0,32 \mathrm{~g} / \mathrm{cm}^{3}$ (B).

\section{DISCUSSÃO}

Os parafusos atuam como elemento de ancoragem dos sistemas de fixação vertebral e as propriedades mecânicas dos sistemas de fixação dependem das suas propriedades mecânicas intrínsecas e da ancoragem dos parafusos nas vértebras ${ }^{(12)}$. A ancoragem dos parafusos nas vértebras está diretamente relacionada, dentre outros fatores secundários, com a densidade mineral do osso, com a técnica de colocação do implante e com o desenho do parafuso ${ }^{(7,13-14)}$.

Os parafusos cônicos foram desenvolvidos para atender às características anatômicas das vértebras e permitir sua ancoragem no osso esponjoso, que predomina e é recoberto por fina camada de osso cortical na sua parte externa ${ }^{(4)}$.

O desenho do parafuso influencia na ancoragem e resistência ao arrancamento do implante, e tem sido 
modificado para aumentar a ancoragem dos parafusos nas vértebras. $\mathrm{O}$ desenvolvimento do parafuso com o diâmetro externo cônico foi a tentativa inicial ${ }^{(15)}$. No entanto, apesar dos bons resultados clínicos com a sua utilização, os parafusos com o desenho externo cônico podem apresentar propriedades mecânicas inferiores aos parafusos cilíndricos ${ }^{(16)}$.

Uma nova modalidade de parafuso foi desenvolvida para aumentar a resistência ao arrancamento, representada por parafusos com a parte externa cilíndrica e com a parte interna (alma do parafuso) cônica. Esses parafusos possuem diâmetro interno que aumenta a partir de sua ponta em direção à cabeça. A introdução do implante com o aumento progressivo de seu diâmetro interno promove a compressão do material ao seu redor, aumentando desse modo sua resistência ao arrancamento $^{(2)}$.

O objetivo do estudo realizado foi comparar esta nova modalidade de parafuso do sistema de fixação vertebral com o tipo de parafuso cilíndrico que tem sido correntemente utilizado, com ênfase para a fixação anterior da coluna vertebral. Outros fatores como a densidade mineral do osso e a técnica de colocação do implante, também foram acrescentados ao desenho experimental do estudo, devido a sua importância no parâmetro estudado.

A resistência ao arrancamento é uma das propriedades mecânicas do parafuso, e é de importância fundamental para a realização das manobras de correção após a aplicação dos implantes. Ela também está relacionada com a falha dos implantes submetidos a cargas cíclicas, situação mais próxima da realidade clínica. Os implantes com menor resistência ao arrancamento têm apresentado maior probabilidade de falha quando submetidos às cargas cíclicas ${ }^{(4,17)}$.

Os resultados observados no estudo que realizamos demonstraram a maior resistência ao arrancamento dos parafusos com a parte interna (alma) cônica. Essa tendência também foi observada em outros estudos indicando que a compressão do tecido ao redor do parafuso, provocada pela introdução do diâmetro interno crescente, pode promover aumento da resistência ao arrancamento do implante ${ }^{(4-5)}$. $\mathrm{O}$ maior torque de in- serção dos parafusos com a parte interna cônica também corrobora essa hipótese.

O desenho do estudo realizado também permitiu demonstrar a importância da densidade do tecido ósseo e do modo de colocação dos parafusos sobre a resistência ao arrancamento dos implantes. A importância desses parâmetros tem sido relatada e foi marcante a observação da alteração do comportamento do mesmo tipo de parafuso frente à alteração das outras variáveis ${ }^{(11,18-25)}$. Esses resultados demonstram que, para a obtenção máxima da propriedade mecânica do implante, relacionada com sua resistência ao arrancamento, não é suficiente a alteração isolada do desenho do parafuso. A utilização de um parafuso com desenho aperfeiçoado pode não resultar em aumento da sua resistência ao arrancamento se as outras variáveis não forem consideradas. O tipo de preparo do orifício-piloto e a densidade do tecido ósseo complementam a propriedade mecânica do desenho do parafuso em relação à sua resistência ao arrancamento e devem ser considerados na aplicação dos implantes vertebrais.

\section{CONCLUSÕES}

O torque de inserção e a resistência ao arrancamento dos parafusos utilizados neste estudo foram influenciados pelo desenho dos parafusos, pela densidade dos corpos de prova e pelo diâmetro do orifício-piloto.

Os parafusos com diâmetro interno cônico apresentaram maior torque de inserção e maior força de arrancamento que os parafusos cilíndricos.

$\mathrm{O}$ diâmetro de perfuração, quando realizado com medida inferior à do diâmetro interno dos parafusos cilíndricos, proporcionou maior força de arrancamento. E, para os parafusos cônicos, a maior resistência ao arrancamento foi observada quando estes foram inseridos em orifícios realizados com medida igual à do menor diâmetro interno.

\section{REFERÊNCIAS}

1. Dwyer AF, Newton NC, Sherwood AA. An anterior approach to scoliosis. A preliminary report. Clin Orthop Relat Res. 1969; (62):192-202.

2. Inceoglu S, Ferrara L, McLain RF. Pedicle screw fixation strength: pullout versus insertional torque. Spine J. 2004;4(5): 513-8. 
3. Hsu CC, Chao CK, Wang JL, Hou SM, Tsai YT, Lin J. Increase of pullout strength of spinal pedicle screws with conical core: biomechanical tests and finite element analyses. J Orthop Res. 2005;23(4):788-94.

4. Lill CA, Schneider E, Goldhahn J, Haslemann A, Zeifang F. Mechanical performance of cylindrical and dual core pedicle screws in calf and human vertebrae. Arch Orthop Trauma Surg. 2006;126(10):686-94.

5. Seller K, Wahl D, Wild A, Krauspe R, Schneider E, Linke B. Pullout strength of anterior spinal instrumentation: a product comparison of seven screws in calf vertebral bodies. Eur Spine J. 2007;16(7):1047-54.

6. Robert KQ 3rd, Chandler R, Baratta RV, Thomas KA, Harris MB. The effect of divergent screw placement on the initial strength of plate-to-bone fixation. J Trauma. 2003;55(6):113944.

7. Daftari TK, Horton WC, Hutton WC. Correlations between screw hole preparation, torque of insertion, and pullout strength for spinal screws. J Spinal Disord. 1994;7(2):139-45.

8. McKinley TO, McLain RF, Yerby SA, Sarigul-Klijn N, Smith TS. The effect of pedicle morphometry on pedicle screw loading. A synthetic model. Spine. 1997;22(3):246-52.

9. McLain RF, McKinley TO, Yerby SA, Smith TS, Sarigul-Klijn $\mathrm{N}$. The effect of bone quality on pedicle screw loading in axial instability. A synthetic model. Spine. 1997;22(13):1454-60.

10. Cunningham BW, Sefter JC, Shono Y, McAfee PC. Static and cyclical biomechanical analysis of pedicle screw spinal constructs. Spine. 1993;18(12):1677-88.

11. Pfeiffer FM, Abernathie DL, Smith DE. A comparison of pullout strength for pedicle screws of different designs: a study using tapped and untapped pilot holes. Spine. 2006;31(23): E867-70.

12. Brantley AG, Mayfield JK, Koeneman JB, Clark KR. The effects of pedicle screw fit. An in vitro study. Spine. 1994; 19(15):1752-8.

13. Cook SD, Salkeld SL, Stanley T, Faciane A, Miller SD. Biomechanical study of pedicle screw fixation in severely osteoporotic bone. Spine J. 2004;4(4):402-8.

14. Kwok AW, Finkelstein JA, Woodside T, Hearn TC, Hu RW. Insertional torque and pull-out strengths of conical and cylindrical pedicle screws in cadaveric bone. Spine. 1996; 21(21):2429-34.

15. Lapresle P, Missenard G. Hydroxylapatite-coated Diapason screws: first clinical report. J Spinal Disord. 1995;8 Suppl 1: S31-9.

16. Lill CA, Schlegel U, Wahl D, Schneider E. Comparison of the in vitro holding strengths of conical and cylindrical pedicle screws in a fully inserted setting and backed out 180 degrees. $\mathrm{J}$ Spinal Disord. 2000;13(3):259-66.

17. McLain RF, Fry MF, Moseley TA, Sharkey NA. Lumbar pedicle screw salvage: pullout testing of three different pedicle screw designs. J Spinal Disord. 1995;8(1):62-8.

18. Alobaid A, Arlet V, Busato A, Steffen T. Pull-out strength of the suprapedicle claw construct: a biomechanical study. Eur Spine J. 2005;14(8):759-64.

19. Coe JD, Warden KE, Herzig MA, McAfee PC. Influence of bone mineral density on the fixation of thoracolumbar implants. A comparative study of transpedicular screws, laminar hooks, and spinous process wires. Spine. 1990;15(9):902-7.

20. Halvorson TL, Kelley LA, Thomas KA, Whitecloud TS 3rd, Cook SD. Effects of bone mineral density on pedicle screw fixation. Spine. 1994;19(21):2415-20.

21. Okuyama K, Abe E, Suzuki T, Tamura Y, Chiba M, Sato K. Can insertional torque predict screw loosening and related failures? An in vivo study of pedicle screw fixation augmenting posterior lumbar interbody fusion. Spine. 2000;25(7):858-64.

22. Papagelopoulos PJ, Currier BL, Stone J, Grabowski JJ, Larson DR, Fisher DR, et al. Biomechanical evaluation of occipital fixation. J Spinal Disord. 2000;13(4):336-44.

23. Reitman CA, Nguyen L, Fogel GR. Biomechanical evaluation of relationship of screw pullout strength, insertional torque, and bone mineral density in the cervical spine. J Spinal Disord Tech. 2004;17(4):306-11.

24. Soshi S, Shiba R, Kondo H, Murota K. An experimental study on transpedicular screw fixation in relation to osteoporosis of the lumbar spine. Spine. 1991;16(11):1335-41.

25. Yamagata M, Kitahara H, Minami S, Takahashi K, Isobe K, Moriya $\mathrm{H}$, et al. Mechanical stability of the pedicle screw fixation systems for the lumbar spine. Spine. 1992;17(3 Suppl): S51-4.
Declaração de inexistência de conflitos de interesse: Não há conflito de interesses na elaboração deste artigo. 